\title{
Crystal Structure of the $\mathrm{N}$-terminal Domain of Anaphase-promoting Complex Subunit $7^{*[5}$
}

Received for publication, June 26, 2008, and in revised form, December 9, 2008 Published, JBC Papers in Press, December 17, 2008, DOI 10.1074/jbc.M804887200

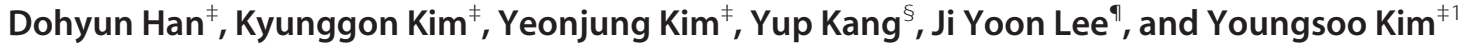 \\ From the ${ }^{\ddagger}$ Department of Biomedical Sciences and Cancer Research Institute, Seoul National University College of Medicine,

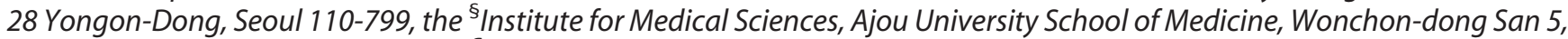 \\ Suwon, Kyunggi-do 442-749, and the "National Instrumentation Center for Environmental Management, Seoul National \\ University, Seoul 151-921, Korea
}

Anaphase-promoting complex or cyclosome $(\mathrm{APC} / \mathrm{C})$ is an unusual E3 ubiquitin ligase and an essential protein that controls mitotic progression. APC/C includes at least 13 subunits, but no structure has been determined for any tetratricopeptide repeat (TPR)-containing subunit (Apc3 and -6-8) in the TPR subcomplex of APC/C. Apc7 is a TPR-containing subunit that exists only in vertebrate $\mathrm{APC} / \mathrm{C}$. Here we report the crystal structure of quad mutant of nApc7 (N-terminal fragment, residues 1-147) of human Apc7 at a resolution of $2.5 \AA$. The structure of $\mathrm{nApc} 7$ adopts a TPR-like motif and has a unique dimerization interface, although the protein does not contain the conserved TPR sequence. Based on the structure of nApc7, in addition to previous experimental findings, we proposed a putative homodimeric structure for full-length Apc7. This model suggests that TPR-containing subunits self-associate and bind to adaptors and substrates via an IR peptide in TPR-containing subunits of $\mathrm{APC} / \mathrm{C}$.

Anaphase-promoting complex/cyclosome $(\mathrm{APC} / \mathrm{C})^{2}$ is an E3 ubiquitin ligase that controls mitotic progression (1). APC/C is an $\sim 1.7$-MDa protein complex that is composed of at least 13 subunits, and it contains a cullin homolog (Apc2), a ring- $\mathrm{H} 2$ finger domain (Apc11), and a tetratricopeptide repeat (TPR)containing subunit (TPR subunit; Apc3 and -6-8) (2). Most TPR subunits are essential and evolutionarily conserved in eukaryotes (3).

$\mathrm{APC} / \mathrm{C}$ requires two adaptors that contain a $\mathrm{C}$-terminal WD40 domain, Cdc20 and Cdh1, to recruit and select various substrates at different stages of the cell cycle. Moreover, both

* This work was supported by the 21C Frontier Functional Proteomics Project of the Korean Ministry of Science and Technology Grant FPR 08-A2-110 and by the Innovative Research Institute for Cell Therapy, Republic of Korea, Grant A062260.

The atomic coordinates and structure factors (code 3FFL) have been deposited in the Protein Data Bank, Research Collaboratory for Structural Bioinformatics, Rutgers University, New Brunswick, NJ (http://www.rcsb.org/).

5 The on-line version of this article (available at http://www.jbc.org) contains supplemental Figs. S1 and S2 and an additional reference.

${ }^{1}$ To whom correspondence should be addressed: Dept. of Biomedical Sciences, Seoul National University College of Medicine, 28 Yongon-Dong, Chongno-Ku, Seoul 110-799, Korea. Tel.: 82-2-740-8073; Fax: 82-2-7410253; E-mail: biolab@snu.ac.kr.

${ }^{2}$ The abbreviations used are: APC/C, anaphase-promoting complex/cyclosome; TPR, tetratricopeptide repeat; CTPR3, consensus TPR number of repeats 3 ; r.m.s.d., root-mean-squared deviation; TLS, translation, libration, and screw-rotation; Apc, anaphase-promoting complex subunit; OGT, O-linked GlcNAc transferase; Sel-Met, selenomethionine; WT, wild type. adaptors and specific $\mathrm{APC} / \mathrm{C}$ subunits contribute to substrate recognition (4).

APC/C specifically ubiquitinates cell cycle regulatory proteins that contain destruction (D) or KEN box motifs (5-7), which target them for destruction by the $26 \mathrm{~S}$ proteosome (8). During the cell cycle, APC/C mediates the metaphase-anaphase transition by ubiquitinating and degrading securin, a separase inhibitor, which participates in the degradation of chromatic cohesion complexes and ubiquitinates B-type cyclin, thereby accelerating transition from the late mitotic phase to $G_{1}(9)$. In addition to its primary role in cell cycle regulation, $\mathrm{APC} / \mathrm{C}$ participates in postmitotic processes, such as regulation of synaptic size and axon growth $(10,11)$.

To assess the mechanism that underlies cell cycle regulation by $\mathrm{APC} / \mathrm{C}$ and the various roles of its subunits, we need to understand how $\mathrm{APC} / \mathrm{C}$ is organized into higher order structures and the manner in which the subunits assemble. Although little is known regarding the crystal structures of APC/C components, three-dimensional models of $\mathrm{APC} / \mathrm{C}$ have recently been obtained by cryo-negative staining EM in human, Xenopus laevis, Saccharomyces cerevisiae, and Schizosaccharomyces pombe (12-15). Several studies have indicated that APC/C assumes an asymmetric triangular shape that is composed of an outer shell and a cavity that extends through its center $(12,14)$. Furthermore, APC/C includes a catalytic subcomplex (Doc1/ Apc10, Apc11, and Apc2), a structural complex (Apc1, Apc4, and Apc5), and a TPR subcomplex (TPR-containing subunits and nonessential subunits) (16).

A TPR unit consists of a 34-residue repeat motif that adopts a helix-turn-helix conformation, which is associated with protein-protein interactions (17). Multiple copies of TPR-containing subunits are organized into the TPR subcomplex within $\mathrm{APC} / \mathrm{C}$, and this subcomplex is functionally important for the recruitment of adaptors and substrates (18). In fact, adaptors (Cdc20 and Cdh1) and Doc1/Apc10 bind to the $\mathrm{C}$-terminal domain of the TPR-containing subunits Apc3 and Apc7 via the IR peptide tail sequence $(7,16$, 19). It is unknown, however, how TPR-containing subunits form homo- and heterosubunit complexes, although studies have demonstrated that TPR-containing subunits self-associate in vivo and in vitro (15) and that they interact with other TPR-containing subunits (20).

Apc7 is found only in vertebrate $\mathrm{APC} / \mathrm{C}$ and is estimated to contain 9-15 TPR motifs, similar to other TPR-containing subunits (9). Apc7 is considered to be a molecular descendant of 
the same ancestral protein that gave rise to Apc3. Furthermore, the $\mathrm{N}$-terminal domain of Apc7 has been reported to contain cell cycle-regulated phosphorylation sites (21), and the C-terminal TPR domain of Apc7 interacts with Cdh1 and Cdc20 (19). In Drosophila APC/C, the homolog of vertebrate Apc7 participates in synergistic genetic interactions with other TPRcontaining subunits (22).

The function of Apc7 within vertebrate $\mathrm{APC} / \mathrm{C}$, however, is poorly understood. Moreover, although the $\mathrm{C}$-terminal regions of Apc3 and Apc7 include a tandem of nine TPR motifs, the $\mathrm{N}$-terminal domains of human Apc3 and Apc7 share little homology with the canonical TPR sequence. Therefore, the $\mathrm{N}$-terminal domain of human Apc7 is expected to have a significant function in vertebrate $\mathrm{APC} / \mathrm{C}$.

In this study, we determined the crystal structure of the $\mathrm{N}$-terminal fragment of human Apc7 (residues 1-147, denoted nApc7), and the homodimeric self-association of nApc7 structure led us to insights into mechanisms of vertebrate APC/C.

\section{EXPERIMENTAL PROCEDURES}

Cloning, Expression, and Purification-The N-terminal fragment of human Apc7 (residues 1-147, nApc7) was amplified by PCR from a plasmid encoding full-length human Apc7 and subcloned into the NdeI-XhoI site of the pET24(+) and pET28(+) vectors. The pET24(+) vector adds a C-terminal His tag to the expressed recombinant protein, whereas pET28(+) adds an $\mathrm{N}$-terminal $\mathrm{His}$ tag and a thrombin cleavage site to the expressed recombinant protein. Apc7 constructs were transformed into Escherichia coli BL21-CodonPlus (DE3)-RIL (Stratagene, Madison, WI).

E. coli BL21-CodonPlus (DE3)-RIL containing the pET28a(+)nApc7 was grown in LB medium containing kanamycin (50 $\mu \mathrm{g} / \mathrm{ml}$ ) overnight at $37^{\circ} \mathrm{C}$. When the absorbance at $600 \mathrm{~nm}$ reached $0.5-0.6$, protein expression was induced by adding 0.5 mM isopropyl D-thiogalactopyranoside at $20{ }^{\circ} \mathrm{C}$. After overnight induction, cells were harvested by centrifugation at 5,000 $\times g$ for $15 \mathrm{~min}$. Cell pellets were resuspended in ice-cold $20 \mathrm{~mm}$ Tris- $\mathrm{HCl}$, pH 7.8, containing $200 \mathrm{~mm} \mathrm{NaCl}, 1 \mathrm{~mm} \beta$-mercaptoethanol, and a protease inhibitor mixture (Roche Applied Science). Resuspended cells were lysed by ultrasonication. Cell supernatants were obtained by centrifugation at $15,000 \times g$ for $50 \mathrm{~min}$ at $4{ }^{\circ} \mathrm{C}$.

Selenomethionine (Sel-Met)-substituted nApc7 protein was expressed according to our protocols, as described previously (23). Additionally, cells were grown at $20{ }^{\circ} \mathrm{C}$ for $90 \mathrm{~min}$ and then induced with isopropyl D-thiogalactopyranoside $(0.5 \mathrm{~mm})$ for $24 \mathrm{~h}$ at $20^{\circ} \mathrm{C}$. Sel-Met-substituted protein was purified using nickel-nitrilotriacetic acid resin (Peptron, Daejeon, Chungnam, South Korea) and a Superdex S-200 gel filtration column. Purified protein was dialyzed into storage buffer $(20 \mathrm{~mm}$ Tris, $\mathrm{pH} 8.0,1 \mathrm{~mm} \beta$-mercaptoethanol, and $0.1 \mathrm{~mm}$ phenylmethylsulfonyl fluoride) and concentrated to $10 \mathrm{mg} / \mathrm{ml}$ by ultrafiltration using an Amicon filter (Millipore, Billerica, MA). Five millimolar 1,4-dithiothreitol was added to all buffers to prevent Sel-Met oxidation. The protocol described above was used to prepare the quad mutant of nApc7 (mnApc7).

Crystallization and Data Collection-Crystals of wild-type (WT) nApc7 protein were obtained at $21^{\circ} \mathrm{C}$ by the sitting-drop vapor diffusion method. The drops consisted of $2.0 \mu \mathrm{l}$ of protein solution (20 mM Tris, $\mathrm{pH} 8.0$ ) mixed with $2.0 \mu$ l of reservoir solution (0.2 M sodium/potassium tartrate, 15\% PEG3350). Droplets were equilibrated against $400 \mu \mathrm{l}$ of reservoir solution, and protein crystals that were $0.1 \times 0.1 \times 0.1 \mathrm{~mm}$ were obtained after 3 days at $21^{\circ} \mathrm{C}$. WT crystals were only diffracted to a resolution of $3 \AA$, but we could not obtain any phasing information. To reduce the number of methionine residues in the asymmetric unit, the methionine residues in WT nApc7 were mutated to other hydrophobic amino acids. Eventually, only the quad mutant (denoted mnApc7; containing M29L/ M108L/M114V/M140L) generated a new crystal form that had enhanced diffraction characteristics, although we tried to crystallize all mutant constructs, i.e. the constructs that contained one, two, or three mutations.

Quad mutant mnApc7 crystals were grown in $200 \mathrm{~mm}$ magnesium formate and $20 \%$ (w/v) PEG3350 at $21^{\circ} \mathrm{C}$. After crystal conditions were optimized, crystals were grown to $0.2 \times 0.2 \times$ $0.1 \mathrm{~mm}$ after 1 week. The final crystallization solutions contained $200 \mathrm{~mm}$ magnesium formate, 15\% (w/v) PEG3350, and $10 \mathrm{~mm}$ strontium chloride. For data collection, crystals were transferred to a cryoprotectant solution (200 mM magnesium formate, $\mathrm{pH} 8.0,20 \%$ (w/v) PEG3350, 10\% glycerol) and flashfrozen in liquid nitrogen at $100 \mathrm{~K}$.

Diffraction data were collected at three wavelengths (0.97950, 0.97956, and $0.97171 \AA$ ) on the 4A beam line at the Pohang Accelerator Laboratory, Republic of Korea. mnApc7 crystals were diffracted to a resolution of $2.5 \AA$. Crystals were found to belong to the $P 2{ }_{1}$ space group, with unit cell dimensions of $a=80.5 \AA, b=64.1 \AA, c=81.6 \AA$ and $\alpha=\gamma=90^{\circ}$, and $\beta=95.2^{\circ}$, and contained four molecules in an asymmetric unit with a Matthews coefficient of $2.6 \AA \mathrm{Da}^{-1}$ (solvent content = 55\%) (24). Data from 50 to $2.5 \AA$ were indexed and scaled using the HKL2000 software package (HKL, Charlottesville, VA). Statistical findings related to data collection and processing are summarized in Table 1.

Structure Determination and Refinement-The overall structure of nApc7 was determined using the optimized multiwavelength anomalous diffraction method (25). To optimize dispersive scattering, we chose a remote energy of $\sim 500 \mathrm{eV}$ away from the edge energy. Sel-Met positions and phasing were determined using SOLVE/RESOLVE $(26,27)$. One molecule contained four selenium sites, and thus a total of 16 selenium sites were present per asymmetric unit. Ten selenium sites were well located by SOLVE. As a result, an auto-traced model was obtained using RESOLVE, in which misconnected loop regions were manually rebuilt with good connectivity.

Initial model building was performed using the "O" program (28). After mutation of the four methionine residues to three leucines and one valine, the residues were fitted into an experimental electron density map. Further model building was carried out using COOT (29). The final model contained residues $1-146$ at a resolution of $2.5 \AA$, without the $20 \mathrm{~N}$-terminal vector residues, the 20 residues of the flexible linker (residues 77-96), and 1 C-terminal residue (residues 147). Interestingly, the inserted residues of the cloning vector, $\mathrm{pET} 28 \mathrm{a}(+)$, were disordered in the experimental electron density map, indicating that 
A
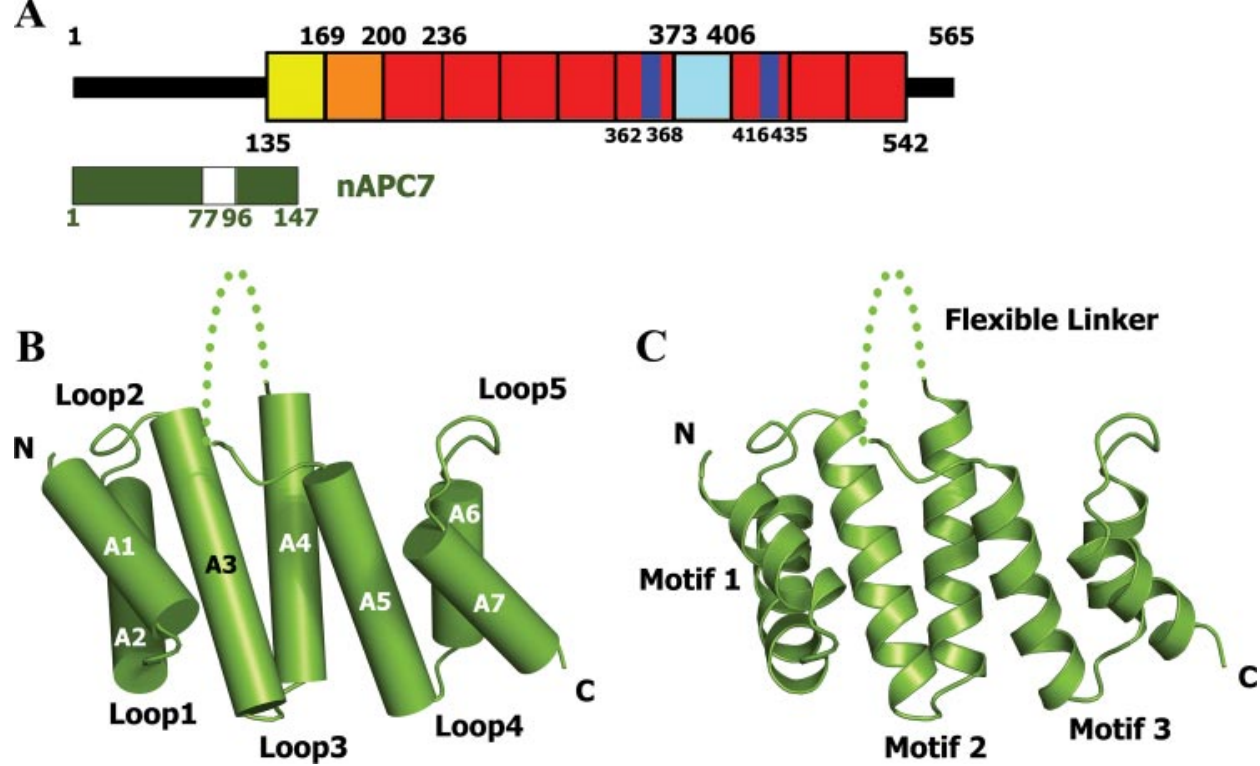

FIGURE 1. Domain configuration of Apc7 and crystal structure of $\mathbf{n A p c 7}$. A, schematic representation of human Apc7. The molecule contains undefined $\mathrm{N}$-terminal regions (residues 1-135), one TPR motif at the $\mathrm{N}$-terminal domain (residues 135-168; yellow box), an undefined region between the $\mathrm{N}$ - and C-terminal domains (residues 169-200; orange box), and the C-terminal domain (residues 200-565) containing nine TPR repeats (consecutive blocks in red and cyan). The sixth TPR motif at the C-terminal domain is colored cyan. The two regions that interact with CBP/P300 are colored deep blue (residues 362-368 and 416-435). nApc7 (residues 1-147, green) is shown below; residues 77-96 form the disordered linker. $B$, structure of nApc7 shown as cylindrical shapes. The dotted line represents a flexible linker (residues 77-96), not modeled in the crystal structure. All helix and loops are labeled as shown. $C$ (or $C^{\prime}$ ) and $N\left(\right.$ or $N^{\prime}$ ) represent the $\mathrm{C}$ and $\mathrm{N}$ termini for each molecule, respectively, in all figures. C, structure of $\mathrm{nApc7}$ as a ribbon diagram. The TPR-like motifs, which consist of two helices, are represented as motifs $1-3$, respectively, starting at the $\mathrm{N}$ terminus.

the nApc7 model did not contain amino acids that originated from the vector.

The model was refined using REFMAC 5 with the Translation, Libration, and Screw-rotation (TLS) option (30). TLS refinement was applied to the final models with TLS groups for each chain that was recommended by the TLSMD server (31). The optimal TLS groups for each chain were composed of three TLS group segments (residues 1-54, 55-113, and 114-146 in monomer A; residues 1-52, 53-116, and 117-146 in monomer B; residues 2-48, 49-104, and 105-146 in monomer C; and residues 2-21, 22-122, and 123-145 in monomer D). The final model was refined to an $R / R_{\text {free }}$ of $0.217 / 0.241$, with 68 water molecules per asymmetric unit, and analyzed using PROCHECK in CCP4i (32). The Ramachandran plot of the nApc7 model revealed that $93.1 \%$ of the residues were in the most favored region, $6.9 \%$ of the residues were in the allowed region, and no residues were in the disallowed region. Refinement statistics are summarized in Table 1.

Site-directed Mutagenesis-After analyzing the secondary structure of Apc7 using the GOR4 program (33), the four methionine residues were substituted with three leucines and one valine to enhance crystallization and reduce Sel-Met content in the asymmetric unit of the crystal. Site-specific mutations (M29L, M108L, M114V, and M108L) in nApc7 were introduced by site-directed mutagenesis PCR. Constructs were confirmed by DNA sequencing. Mutants were expressed and purified using the protocol described above for the wild-type protein.
Analytical Gel Filtration-Chromatography was performed using a Superdex-200 Tricorn 10/300 column attached to an AKTA FPLC system (Applied Biosystems, Foster City, CA) at a flow rate of 0.5 $\mathrm{ml} / \mathrm{min}$ using $150 \mu \mathrm{l}$ of protein at a concentration of $1 \mathrm{mg} / \mathrm{ml}$. Protein elution was monitored at $280 \mathrm{~nm}$ using the UNICORN 5.0 program (Applied Biosystems). The column was calibrated with standard proteins, i.e. alcohol dehydrogenase $(150 \mathrm{kDa})$, bovine serum albumin $(67 \mathrm{kDa})$, ovalbumin $(43 \mathrm{kDa})$, and ribonuclease A (15.7 kDa).

Structural Analysis-Structural similarities between nApc7 and known structures were searched for in the SSM server (34). The PISA server was used to identify the dimer interface of the crystal lattice (35). Residues 169-200 were predicted to be a disordered domain linker by DLP-SVM (36). Sequence alignment was carried out using ClustalW (37). The representations rendered were generated using PyMOL (DeLano Scientific LLC, South San Francisco, CA).

\section{RESULTS}

Structure Determination-Human Apc7 has a molecular mass of $62 \mathrm{kDa}$ and is composed of 10 TPR repeats and two primary unassigned regions (residues 1-134 and residues 169200) (Fig. 1A). To obtain insights into the function of the two unassigned regions, various cloning constructs were examined, and consequently, only construct residues 1-147 (nApc7) were able to be expressed as a soluble protein and used for crystallization. This strategy led to the successful crystallization of nApc7, and its structure was determined at a resolution of $2.5 \AA$.

Crystallized nApc7 diffracted at a resolution of 3.0 A. Diffraction data for $\mathrm{nApc7}$ crystals indicated that it belongs to the primitive tetragonal space group, with unit-cell parameters $a=$ 98.7, $b=98.7, c=286.3 \AA$ and $\alpha=\beta=\gamma=90^{\circ}$, and has six molecules per asymmetric unit. We observed many methionine residues $(n=48)$ in the asymmetric unit, which were presumed to interfere with correct phasing. To reduce the methionine count in the asymmetric unit, four methionines (Met-29, Met108, Met-140, and Met-114) were sequentially replaced in nApc7 with three leucines and one valine.

Although we attempted to crystallize all of the mutant constructs (i.e. species that had one, two, three, or four mutations), only the quad mutant (mnApc7; M29L/M108L/M114V/ M140L) generated a new crystal form that harbored enhanced diffraction properties. This new mutant crystal belonged to space group $P 2_{1}$, with cell dimensions of $a=80.5 \AA, b=64.1 \AA$, $c=81.6 \AA$ and $\alpha=\gamma=90^{\circ}$, and $\beta=95.2^{\circ}$, and had four 


\section{Crystal Structure of the N-terminal Domain of Apc7}

\section{TABLE 1}

Data collection and refinement statistics

Data in parentheses are for the highest resolution shell.

\begin{tabular}{|c|c|c|c|}
\hline & \multicolumn{3}{|c|}{ Sel-MetmnApc7 } \\
\hline \multicolumn{4}{|l|}{ Data collection } \\
\hline \multirow{3}{*}{$\begin{array}{l}\text { Space group } \\
\text { Cell dimensions }\end{array}$} & \multirow{2}{*}{\multicolumn{3}{|c|}{$a=80.5, b=64.1, c=81.6 \AA$}} \\
\hline & & & \\
\hline & Peak & Inflection & Remote \\
\hline Wavelength $(\AA)$ & 0.97950 & 0.97956 & 0.97171 \\
\hline Resolution $(\AA)$ & $50 \sim 2.5(2.59-2.50)$ & $50 \sim 2.5(2.59-2.50)$ & $50 \sim 2.5(2.59-2.50)$ \\
\hline$R_{\mathrm{sum}}(\%)$ & $6.7(51.1)$ & $6.3(48.1)$ & $6.3(59.0)$ \\
\hline$I / \sigma I$ & $23.6(1.3)$ & $24.3(1.6)$ & $19.2(1.2)$ \\
\hline Completeness (\%) & $95.4(77.2)$ & $96.0(81.9)$ & $94.6(73.7)$ \\
\hline Redundancy & 5.3 & 5.2 & 5.2 \\
\hline \multicolumn{4}{|l|}{ Refinement } \\
\hline Resolution & & $41.0-2.5 \AA$ & \\
\hline No. of reflections & & 24,411 & \\
\hline$R_{\text {work }} / R_{\text {free }}$ & & $21.4 / 24.1 \%$ & \\
\hline \multicolumn{4}{|l|}{ No. of atoms } \\
\hline Protein & & 3997 & \\
\hline Water & & 48 & \\
\hline \multicolumn{4}{|l|}{$B$-factors } \\
\hline Protein & & $26.8 \AA^{2}$ & \\
\hline Water & & $52.7 \AA^{2}$ & \\
\hline \multirow{2}{*}{\multicolumn{4}{|c|}{ r.m.s.d. }} \\
\hline Bond length & & & \\
\hline Bond angle & & $0.822^{\circ}$ & \\
\hline
\end{tabular}

mnApc7 molecules per asymmetric unit. By mutating the aforementioned four methionines per mnApc7 (for a total of 16 methionines in the asymmetric unit), we overcame the phasing problem. The resultant electron density map from the multiwavelength anomalous diffraction phasing was readily traceable (see supplemental Fig. 1). The final structure was refined to an $R$ value of $21.4 \%$ and an $R_{\text {free }}$ of $24.1 \%$ at a resolution of $2.5 \AA$ (Table 1 ).

Overall Structure of the N-terminal Fragment of Apc7-The overall structure of nApc7 contains seven anti-parallel $\alpha$-helices (A1-A7) and is $41 \AA$ long and $25 \AA$ wide, with a pitch of 30 $\AA$. Each monomer consists of two small domains, comprising residues $1-76$ and $97-146$, which are joined by a disordered flexible linker (residues 77-96) (Fig. 1, $A-C$ ).

The asymmetric unit of the crystal lattice contains four molecules of nApc7 (molecules A-D). The superposition of C- $\alpha$ atoms of the four monomers of $\mathrm{nApc} 7$ in the asymmetric unit yields an r.m.s.d. of $\sim 1 \AA$ onto their corresponding C- $\alpha$ positions. Monomer A forms a tightly packed dimer with its counterpart (monomer $\mathrm{C}^{\prime}$ ) in the neighboring asymmetric unit, in which these two nApc7 molecules are related by the crystallographic 2-fold axis (data not shown; see Fig. $4 A$ ).

Using an SSM server, we searched for known proteins that were structurally similar to nApc7 (34). To this end, the overall folding of $\mathrm{nApc} 7$ resembled that of TPR proteins that contained three TPR repeats, although nApc7 does not contain any sequence that is homologous to the canonical TPR motif. CTPR3 (38) and NrfG (23) proteins, which contain three TPR motifs, were superimposed onto nApc7 using r.m.s.d. values of 1.57 and $2.8 \AA$, respectively, indicating that the TPR-like fold of nApc7 is structurally conserved, despite its lacking the canonical TPR sequence (Trp-4, Leu-7, Gly-8, Tyr-11, Ala-20, Phe24, Ala-27, and Pro-32 of the 34-residue TPR motif) (Fig. 2, $A$ and $B$ ). Interestingly, the insertion of a long flexible linker (residues 77-96) did not disrupt the superhelical shape of the three TPR-like repeats of nApc7 (Fig. 2, $A$ and $B$ ).
nApc7 Folds into Atypical TPR-like Motifs-To compare the TPR-like folds of nApc7 with the typical TPR motif, nApc7 was superimposed onto the TPR domain of CTPR3 (Protein Data Bank code 1NA0), which was the TPR protein that had the highest Z-score, according to a Dali data base server (39). Seven anti-parallel $\alpha$-helices (A1-A7) of nApc7 represented 3.5 tandem TPR-like motifs despite the lack of a conserved canonical sequence (Fig. 2, $A$ and $B$ ).

Although the overall structure of nApc7 assumed a superhelical TPR-like fold, our structural alignment indicated that the structure and conserved sequences of TPR-like motifs differed from those of the typical TPR motif, except for the presence of hydrophobic residues in the A4 helix (Fig. 2B). In particular, the Trp residue at position 4 of the canonical TPR sequence, which generally stabilizes the turns between helices through hydrophobic interactions, does not exist at the corresponding position in the TPR-like motifs of nApc7. Generally, packing interactions between adjacent TPR motifs are mediated by conserved hydrophobic residues in the typical TPR motif.

In contrast, polar interactions, formed primarily by charged amino acids in helices A3 and A5, stabilize packing between TPR-like motifs in nApc7 (Fig. 2C). For example, turn 2 is stabilized by hydrogen bond formation between the side chain of Lys-42 in the A3 helix and the backbone oxygen of Pro-34 in loop 2. Also, packing between TPR-like motif 2 and TPR-like motif 3 is stabilized by polar interactions in both the convex and concave sides; Asn-51 and Lys-107 reinforce packing on the convex side, whereas polar interactions are formed by Arg-60, Lys-74, Glu-101, Lys-105, and Gln-116 on the concave side (Fig. $2 C)$. These charged residues that are involved in packing interactions are highly conserved between other homologous Apc7 subunits (Fig. 3).

Consequently, hydrogen bonds maintain the packing angle between the helices of nApc7 despite its unusually sized helices and loops. Thus, we found that nApc7 has secondary structural 
A

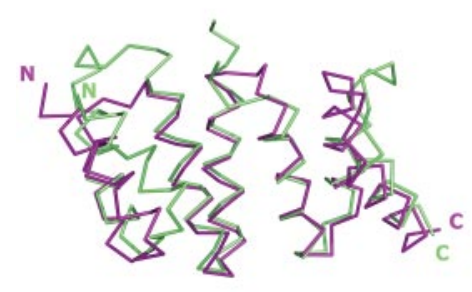

B

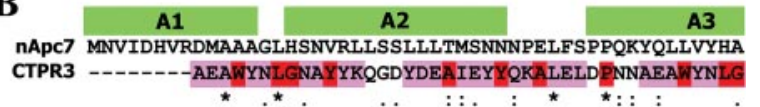

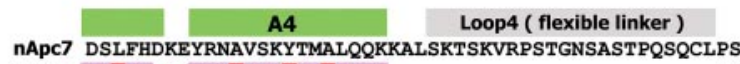

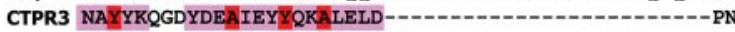
$::::::^{\star}::^{\star}: \star^{\star}{ }^{\star}:$.

\section{A5}

nApC7 EIEVKYKMAECYTMLKQDKDAIAILDGIPSRQRT-PKIMMMLANLYKK CTPR3 NAEAWYNLGNAYYKQGDYDEAIEYYQKALELDPNNAEAWYNLGNAYYK $: \star *^{\star}::, .^{\star}$

C
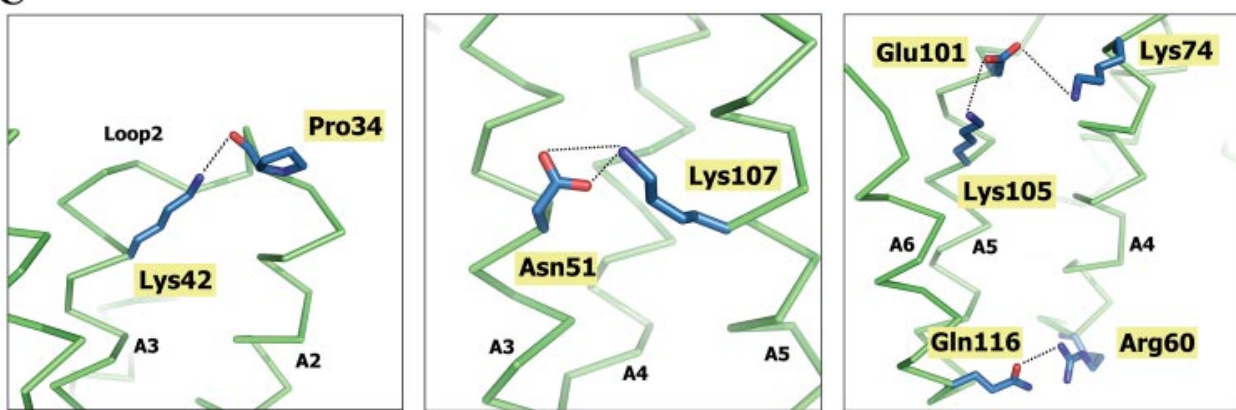

FIGURE 2. Distinct structural features of $\mathbf{n A p c 7}$. A, superimposition of $\mathrm{nApc7}$ onto CTPR3. $\mathrm{nApC7}$ and CTPR are shown in green and pink, respectively. $B$, sequence alignment between nApc7 and CTPR3 (Protein Data Bank code 1NA0). The canonical TPR sequences of CTPR3 are highlighted in red on the bottom line; pink boxes indicate helices. Identical residues are indicated as asterisks, and conserved residues as dots or colons. C, hydrophilic interactions involved in the packing of TPR-like motifs in the structure of nApc7. Dotted lines represent hydrogen bonds, and helices are labeled (e.g. " $A 2$ " is helix 2).

Dimerization interface 1 - 37

Hs

$\mathrm{XI}$

Am

Vv

At

Hs

$\mathrm{x} 1$

Am

$\mathrm{Vv}$

At

Hs

$\mathrm{X} 1$

Am

Vv

At

Hs

$\mathrm{X} 1$

Am

Vv

At

FIGURE 3. Multiple sequence alignments of Apc7 homologs. Multiple sequence alignments of Apc subunits are from Homo sapiens $(H s)$, Xenopus laevis $(X I)$, Apis mellifera $(A m)$, Vitis vinifera $(V v)$, and Arabidopsis thaliana (At). The dimerization interface region, the flexible linker in $\mathrm{nApc7}$, and two predicted TPR motifs are marked by boxes. The undefined flexible linker between the $\mathrm{N}$ - and $\mathrm{C}$-terminal domains is shown at residues 169-200. The residues involved in the hydrophobic interactions that are required for dimerization in the "dimerization interface 1-37" box are blocked in gray and indicated as black stars, whereas the key residues that are involved in the hydrophilic packing of TPR-like motifs also are blocked in gray and indicated as black circles.

features that are unlike those of the typical TPR motif but that its overall shape resembles that of the TPR protein, i.e. a helixloop-helix conformation and a superhelical structure.
$N$-terminal Region of the TPR-like Repeats of $n A p c 7$ Mediates Selfdimerization-The crystal structure of nApc7 revealed it to be a homodimer in which two molecules of nApc7 associate along the crystallographic 2-fold screw axis (data not shown; see Fig. $4 A$ ). The PISA server was used to identify the dimer interface (35). The solventaccessible surface that was calculated between the two symmetry mates was $586 \AA^{2}$, which indicates a relatively small interfacial area. However, the $\Delta^{\mathrm{i}} G$ and $\Delta^{\mathrm{i}} G p$ values that were calculated using the PISA server were $-13.9 \mathrm{kcal} / \mathrm{mol}$ and 0.022 , respectively, which implies that a stable interaction underlies formation of the homodimer.

Two nApc7 monomers are organized in an anti-parallel fashion to form the dimer. Helices A1 and A2 of one monomer interact with the interdigitating helices (A1 and A2) of the other monomer (Figs. $4 A$ and $1 B)$. Overlooking the 2-fold crystallographic axis, the dimer maintains an elongated shape, with overall dimensions of $25 \times 30 \times 80 \AA$ (Figs. $4 A$ and $1 C$ ). Formation of the nApc7 homodimer is mediated by motif 1 (Figs. $4 A$ and $1 C$ ). One face of motif 1 is composed of many hydrophobic side chains that interact with those in the other monomer of the dimer (Fig. 4B). Val-3, His-6, and Met-10 (in the A1 helix); Leu-15 (in loop 1); Leu-21, Leu-22, Leu-25, Leu-26, and Met-29 (in the A2 helix); and Leu-36 and Phe-37 (in loop 2) participate in hydrophobic interactions with the residues of a symmetrically paired molecule (Fig. 4C).

Importantly, sequence alignment showed that the hydrophobic residues that are involved in dimerization are highly conserved between homologous Apc7 subunits (Fig. 3), which suggests that Apc7 dimerization is a common feature of these homologs. Analytical gel filtration showed that nApc7 eluted at a molecular mass of $\sim 34 \mathrm{kDa}$, corresponding to dimeric nApc7 (Fig. $4 D)$. In this study, we mutated two hydrophobic residues that are involved in forming the dimeric interface to charged residues (L22R and L26K) and performed size-exclusion chroma- 
A

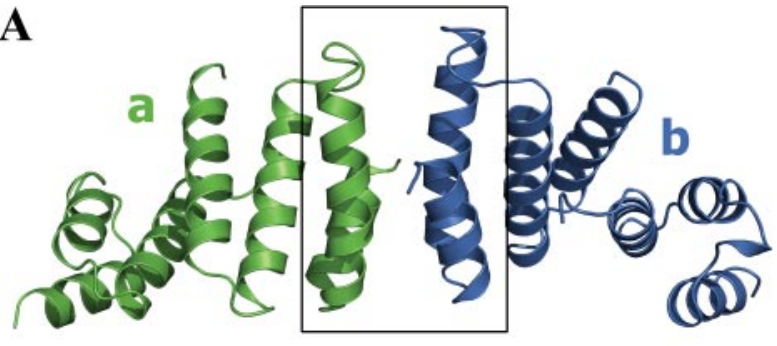

B

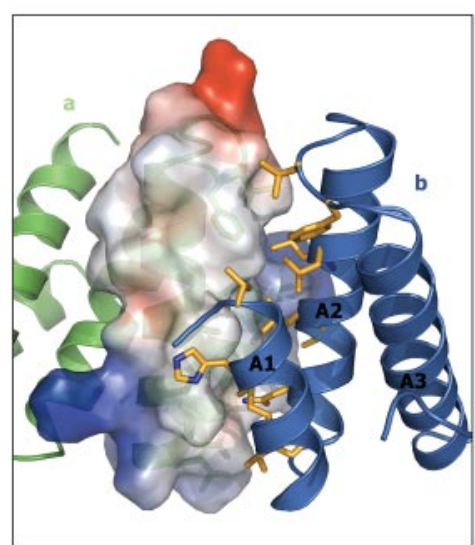

C

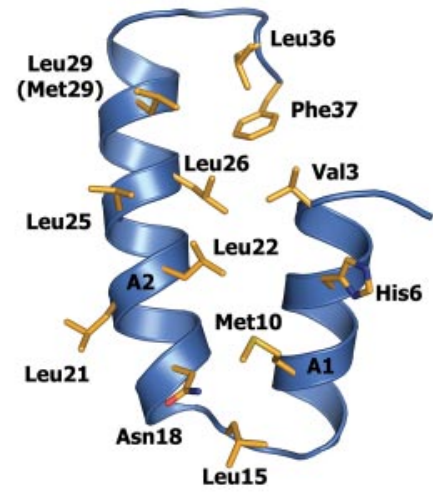

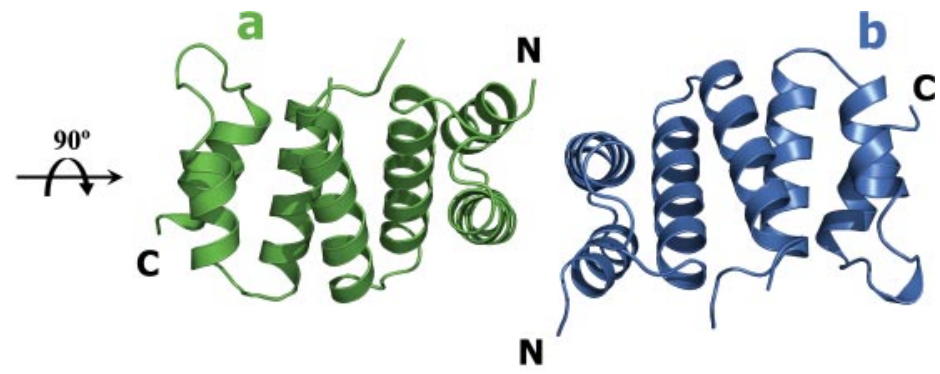

D

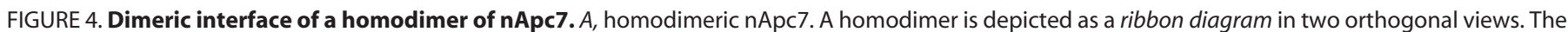

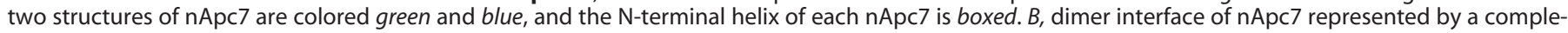

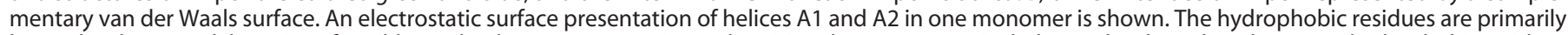

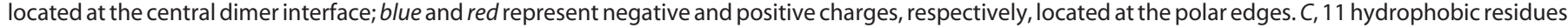

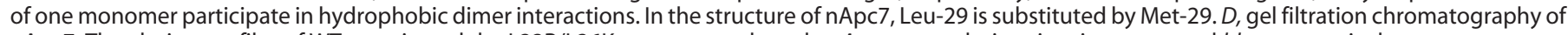
$\mathrm{nApc7}$. The elution profiles of WT protein and the L22R/L26K mutant are plotted as $A_{280}$ versus elution time in orange and blue, respectively.

tography for the double mutant protein. Doubly mutated nApc7 eluted at an apparent monomer size of $17 \mathrm{kDa}$ (Fig. 4D), which supports our hypothesis that the Apc7 subunit exists as a dimer in solution.

Interestingly, two monomers of dimeric nApc7 stack headto-head because of strong hydrophobic interactions, resembling the interaction between adjacent TPR repeats in typical TPR proteins. These distinctive properties of dimerization between the $\mathrm{N}$-terminal regions in TPR and TPR-like proteins were identified for the first time in this study.

Comparison of Modes of Self-association of $n A p c 7$ Versus Other TPR Proteins-Intermolecular TPR domain interactions that result in self-association have been reported for several TPR proteins. To characterize the dimerization of nApc7, we compared the self-association of TPR-like repeats of nApc7 with homodimerization mechanisms of other TPR proteins, such as OGT (40) and Tom70p (translocase of outer membrane complex subunit) (41). Dimer interactions of three TPR proteins (nApc7, OGT, and Tom70p) are mediated by conserved hydrophobic residues. Many hydrophobic residues of nApc7 (11 residues), OGT (4 residues), and Tom70p (14 residues) are involved in dimer formation (Fig. $5 \mathrm{~A}$ ). Hydrophobic contacts between the helices of TPR motifs are a common feature of dimer interfaces in TPR proteins.

In contrast, we observed important conformational differences in the dimerization mode of three proteins (nApc7, OGT, and Tom70p). For example, the dimer interface of OGT comprises B helices of the sixth and seventh TPR motifs that are centered at the local dyad axis of homodimeric OGT (40) (Fig. $5, A$ and $B$ ). For Tom70p, dimerization is mediated by hydrophobic interactions between the $\mathrm{C}$-terminal region (helices $\mathrm{A} 6$ and A7) of the N-terminal TPR domain and the C-terminal region (helices $\mathrm{A} 25$ and $\mathrm{A} 26$ ) of the $\mathrm{C}$-terminal TPR domain (41) (Fig. 5, $A$ and B). Notably, dimerization of nApc7 occurs through hydrophobic interactions between the $\mathrm{N}$-terminal ends of two monomers (helices A1 and A2 of each monomer) (Fig. 5, $A$ and $B$ ).

Domain Flexibility of nApc7 Monomer-Each monomer of nApc7 consists of two domains, which are connected by a long flexible linker. The flexibility of nApc7 can be described by considering the $B$-factors that are obtained by protein crystallography, which characterizes not only their thermal dynamics but also the lack of static ordering in the crystal packing. To this end, isotropic $B$-factors of four monomers in the asymmetric unit were plotted to compare the regions that have high $B$-factors (especially, higher than $60 \AA^{2}$ ) with the corresponding secondary structures of nApc7 (Fig. 6A). Except for the $\mathrm{N}$-terminal and C-terminal ends of the protein, the equivalent locations that have high $B$-factors in the structure primarily are solventexposed loop regions in the helix-turn-helix conformation (Fig. 6A).

Interestingly, significant differences in isotropic $B$-factors between monomers were observed in the $\mathrm{C}$-terminal domain (helix A6, loop 5, and helix A7) (Fig. 6A). In particular, the isotropic $B$-factors of the $C$-terminal region of monomer $\mathrm{D}$ were higher than the corresponding regions in the other three 
A

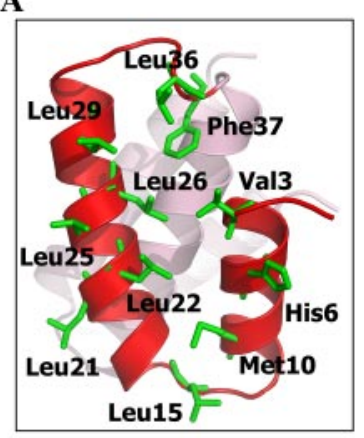

nApc7

B

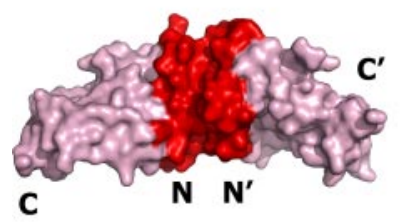

nApc7

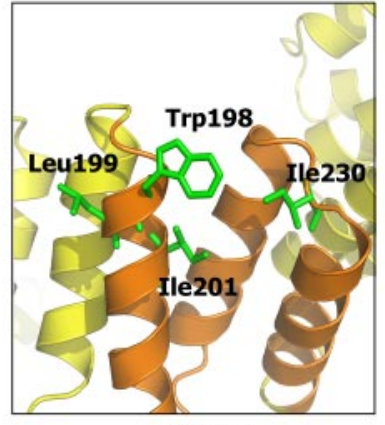

OGT

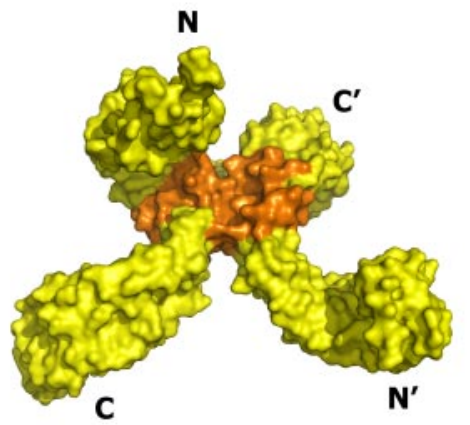

OGT

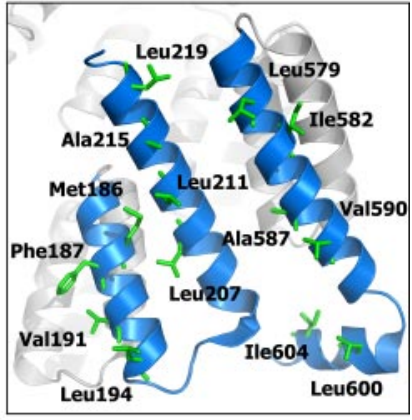

Tom70p

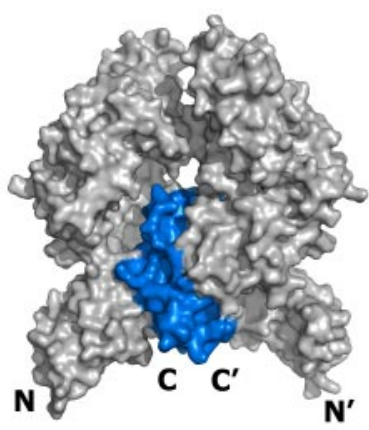

Tom70p
FIGURE 5. Comparison of dimer interface and self-association for three TPR domains. $A$, dimerization interfaces of three proteins in ribbon presentation. The interface is characterized by several hydrophobic residues that are involved in hydrophobic contact. All hydrophobic residues are noted, and the side chains are colored in green. $B$, dimeric conformations of the three proteins shown by surface presentation. The contact regions that are involved in dimerization are shown in different colors ( $\mathrm{nApc7}$, red; OGT, orange; Tom70p, blue). The $\mathrm{N}$ - and C-terminal ends of one molecule of a dimer are labeled as $N$ and $C$, respectively, and those of the other monomer are represented as $N^{\prime}$ and $C^{\prime}$.

monomers ( $\mathrm{A}, \mathrm{B}$, and $\mathrm{C}$ monomers). This outstanding variation in monomer $\mathrm{D}$ led us to believe that the $\mathrm{C}$-terminal domain of nApc7 is much more flexible than the other regions of this molecule in solution.

In addition, because such high average $B$-factors of $\sim 60 \AA^{2}$ were obtained from isotropic $B$-factor refinement for each monomer, we performed additional $B$-factor refinement using TLS group partitioning and motion analysis, where TLS refinement calculates the anisotropic translational and rotational displacements of the partitioned components. After TLS refinement using three TLS groups per monomer (see "Experimental Procedures"), the average $B$-factor of nApc7 decreased to $27 \AA^{2}$ (Fig. 6B). According to a plot of the least squares residual shown by TLSMD (31), the most rapid decline of $B$-factors occurred in the C-terminal region of monomer D, compared with monomers A-C. Apparently, the most significant difference in $B$-factors exists in the $C$-terminal region of monomer $D$ even after $B$-factor refinement using TLS groups (Fig. 6B).

Model of the Human Apc7 Subunit-To establish a model of the intact structure of human Apc7 based on the structure of nApc7, we referred to the TPR domain of OGT (40) and Tom70p (41), because OGT and Tom70p contains a homodimeric TPR structure. Because residues 135-147 of nApc7 were predicted to contain half of one typical TPR motif (Fig. 1A), the other half (residues 148-168) also was modeled

\section{DISCUSSION}

into one TPR motif (residues 135168, the yellow-colored block in Figs. $1 A$ and 7).

On the other hand, the $\mathrm{N}$ - and C-terminal TPR domains of Tom70p are separated by a central flexible linker (41). Similar to Tom70p, Apc7 also contains a defined sequence (residues 169200) between the $\mathrm{N}$ - and $\mathrm{C}$-terminal TPR domains that was predicted to be a disordered domain by DLPSVM (36). It is conceivable that the $\mathrm{N}$-terminal TPR-like domain (residues 1-168, including nApc7, Fig. $1 A$ ) is attached to the nine-TPRcontaining $\mathrm{C}$-terminal TPR domain (residues 201-565) via a flexible linker (residues 169-200) (Figs. 7 and $1 A$ ). The $\mathrm{C}$-terminal domain that contains nine TPR repeats is modeled after those of the structure of OGT (Protein Data Bank code 1W3B). Taken together, the C-terminal TPR domain (containing nine TPR repeats; residues 201-565) and the N-terminal TPR domain (containing four TPR-like repeats; residues 1-168), constituting fulllength Apc7, could be modeled to maintain an overall superhelical conformation (Fig. 7).

In fact, the continuous superhelical pattern of full-length Apc7 may depend on the conformation of the predicted flexible linker (residues 169-200, the dotted loop in the middle in Fig. 7). For example, the tight hydrophobic interactions between the C-terminal helix of the $\mathrm{C}$-terminal TPR domain and the $\mathrm{N}$-terminal helix of the $\mathrm{N}$-terminal TPR domain could generate a continuous superhelix of full-length Apc7, as represented in Fig. 7; otherwise, the two TPR domains can swing easily around each other.

This study reports the crystal structure of the N-terminal domain of human Apc7 (nApc7) and describes the structure of a TPR-containing subunit of APC/C. After numerous attempts at protein engineering and crystallization, we successfully crystallized nApc7 protein and determined its crystal structure. Despite the presence of a long flexible linker (residues 77-96) and a lack of canonical sequence homology, the overall structure of nApc7 resembled that of a typical TPR domain.

The detailed structural features of nApc7, however, differ from those of typical TPR proteins, primarily with regard to its hydrophilic residues, which participate in TPR-like motif packing. Two nApc7 monomers were packed as a dimer with 2-fold symmetry in the crystal. Moreover, nApc7 was found to form a dimer in solution. This homodimerization via its $\mathrm{N}$-terminal dimerization motif is the first example of homodimerization 
A

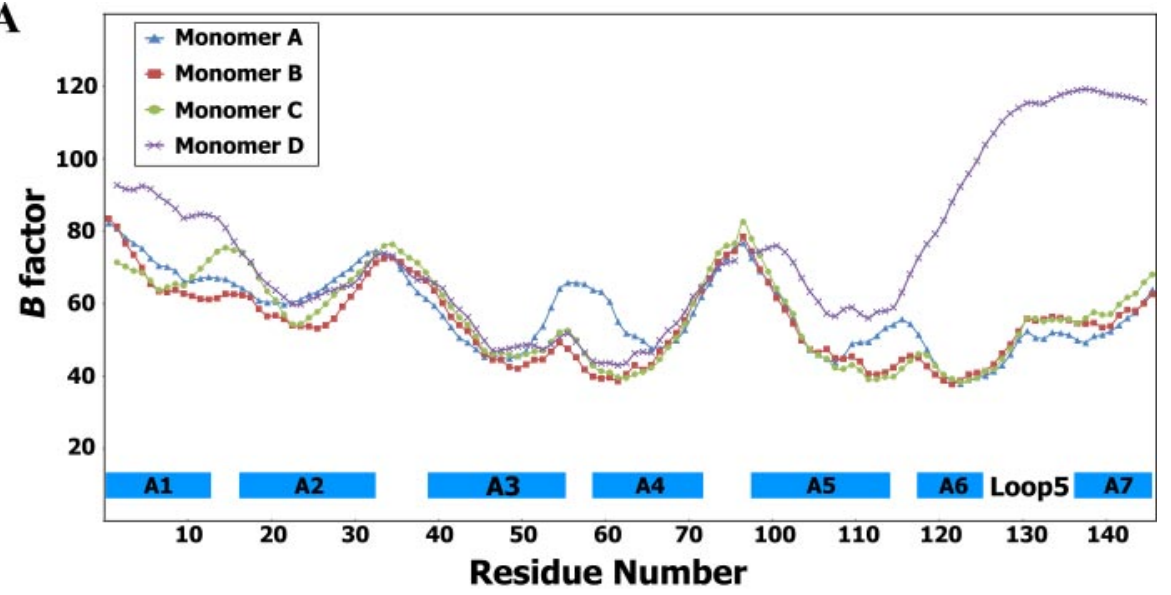

B

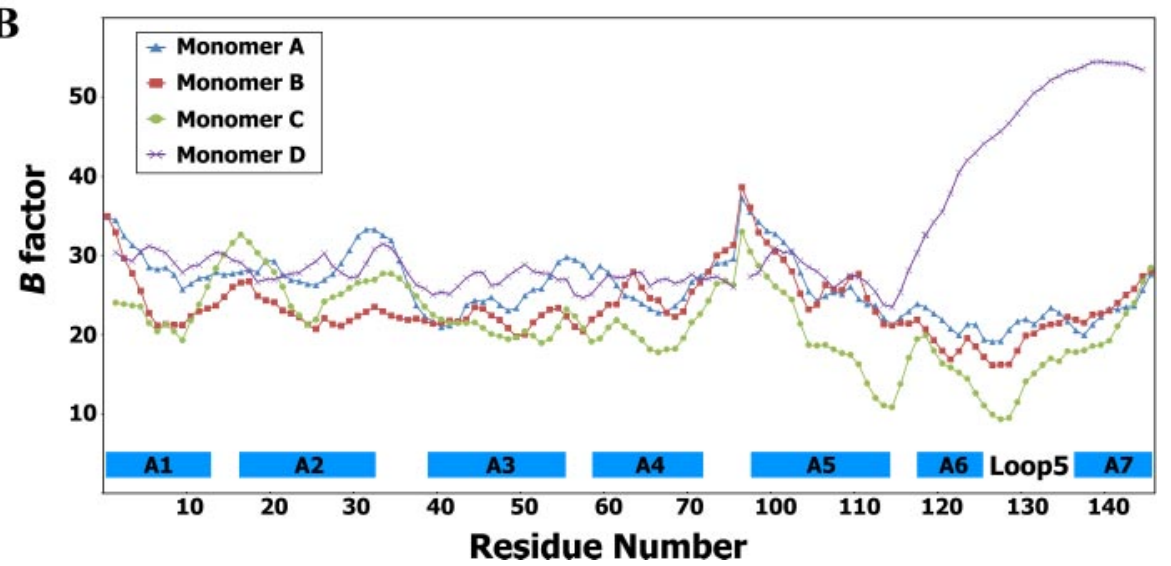

FIGURE 6. B-factor analysis for four monomers in an asymmetric unit. $A$, plot of isotropic $B$-factors for four monomers before TLS refinement. $B$-factor patterns for the four monomers indicate that the $C$-terminal region (helix A6, loop 5, and helix A7) is more flexible than other parts of $n A p c 7$, especially in monomer D. B, plot of $B$-factors after refinement using TLS groups. The secondary structure of nApc7 is shown in the blue box. The $B$-factors of helix A6, loop 5 , and helix A7 are again prominent in the plot of monomer D.
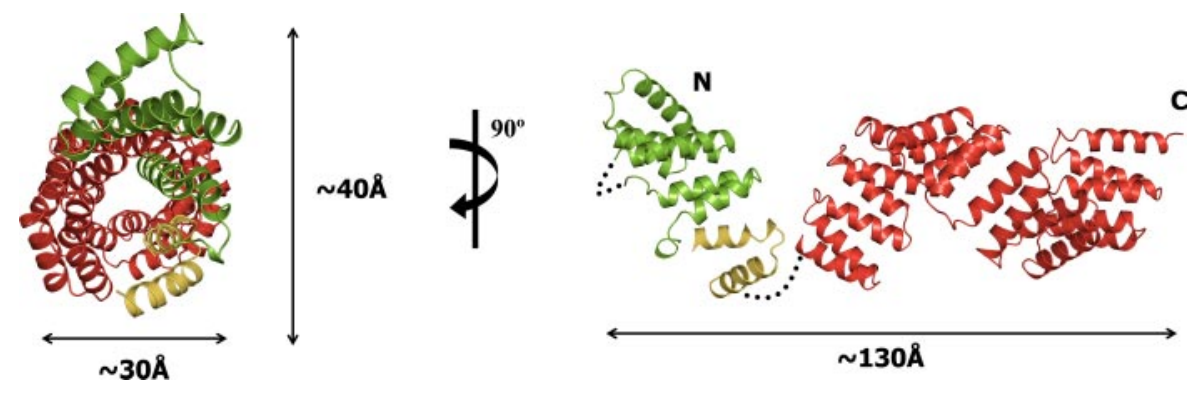

FIGURE 7. Model of full-length human Apc7. A model of full-length Apc7 is shown in two different views (the second image is rotated about the vertical axis $90^{\circ}$ ). Nine TPR motifs, colored in red, at the C-terminal TPR domain were reconstructed based on the 10 TPR motifs of OGT. One TPR motif at the N-terminal TPR domain (residues 135-168) is shown in yellow. Residues 169-200 in the dotted line represent the predicted disordered linker between the $\mathrm{N}$ - and C-terminal TPR domains. The distance between the two TPR domains can be adjusted depending on the conformation of the linker (residues 169-200), which may determine the overall conformation of full-length Apc7.

that is mediated by the $\mathrm{N}$ terminus of TPR protein, although the TPR motif is known to mediate self-association for many TPRcontaining proteins, such as OGT (40), Tom70p (41), and SGT (42).

A comparison of $B$-factors between the four monomers in the asymmetric unit shows that the $\mathrm{C}$-terminal region (helix A6, helix A7, and loop 5) of nApc7 is flexible. Notably, loop 5 has the largest $B$-factors, and in addition to these $\mathrm{B}$-factors, the disorder of residues $169-200$ that is predicted by DLP-SVM
(36) suggests that the middle domain between the $\mathrm{N}$-terminal dimerization domain (residues 1-37) and the C-terminal TPR domain (residues 201-565) acts as a hinge region within full-length Apc7.

Two copies of Apc7 are present per molecule of $\mathrm{APC} / \mathrm{C}$, as are other TPR subunits (Apc3, Apc6, and Apc8) (13), and interact with Apc3, Apc6, and Apc8 in vitro $(22,43)$. Additionally, Apc7 is highly related to Apc3 at the primary sequence level. The N-terminal third of Apc3 includes a single TPR motif and a unique non-TPR segment (20), which is configured similarly to the N-terminal domain of Apc7. Sequence analysis and alignment indicated that the N-terminal nonTPR segment of Apc3 has a hydrophobic surface, like the $\mathrm{N}$-terminal domain of Apc7 (see supplemental Fig. 2). TPR-containing subunits in $\mathrm{APC} / \mathrm{C}$ are predicted to carry out similar functions, i.e. protein-protein interaction and structural unit, via homo- and heteroassociations that involve their common TPR repeats, and these associations appear to be necessary to form the TPR subcomplex. The lack of a structure of TPR-containing subunits in $\mathrm{APC} / \mathrm{C}$, however, has prevented our understanding of their assembly and function.

To gain insight into of the mechanism by which the TPR-containing subunits of $\mathrm{APC} / \mathrm{C}$ associate, we constructed a model structure of full-length Apc7 based on nApc7 and the TPR-repeat domains of OGT (40). In this model, homodimerization of full-length Apc7 is mediated by interactions between the N-terminal dimerization domains, to which we could apply the flexible, disordered middle region (residues 169-200, the dotted loop in the middle of Fig. 7). The conformation of this flexible region, between the $\mathrm{N}$ - and C-terminal TPR domains, may drive homodimerization either in an elongated form or a curved U-shape (Fig. 8, $A$ and $B$ ).

Interestingly, immuno-EM models of Apc3 and Apc8 show that the two $\mathrm{C}$-terminal tails of each subunit are in close proximity on the outer surface of APC/C $(14,15)$. This experimental evidence indicates that the C-terminal domains of TPR-containing subunits (Apc3 and also the homolog Apc7) lie side by 
$\mathbf{A}$
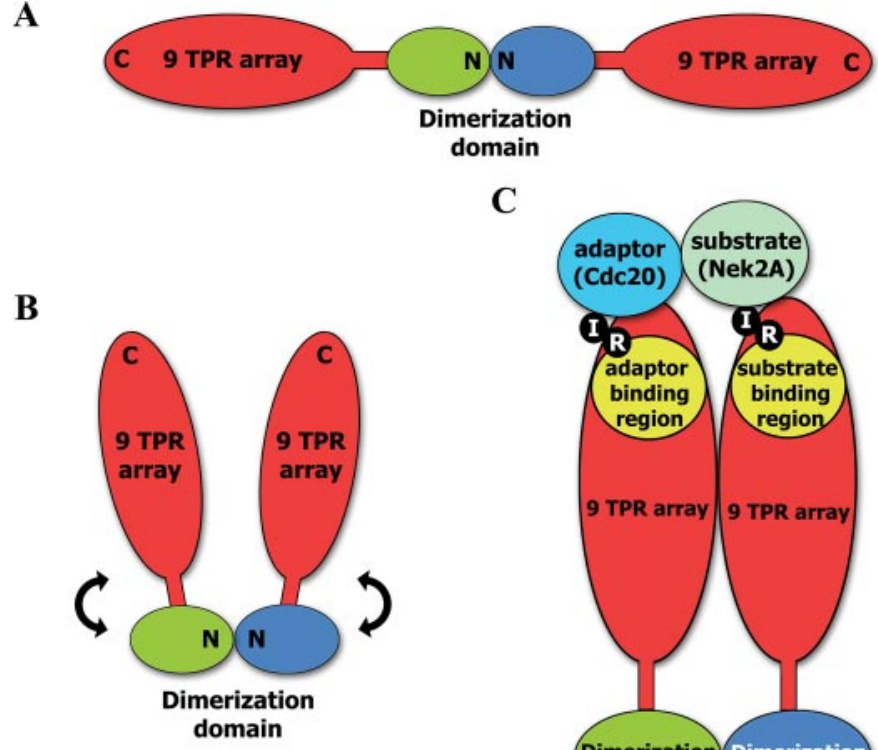

C

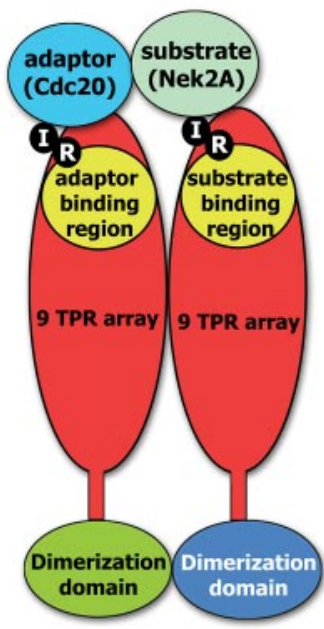

FIGURE 8. Putative models of dimers of full-length Apc7. Putative models depicting two types of dimer configurations. A, elongated model of dimeric Apc7. B, curved U-shaped dimeric Apc7. C, schematics of the putative Apc7 dimer, illustrating how adaptor and substrate proteins can simultaneously bind to the C-terminal TPR domain via IR peptides. The small and big lobes represent the $\mathrm{N}$ - and $\mathrm{C}$-terminal TPR domains, respectively.

side and that the C-terminal TPR domains are oriented toward the outer surface.

Moreover, based on a sequence alignment of Apc7 and Apc3, the sixth TPR (residues 373-406) of the C-terminal TPR domain of Apc7 corresponds to the seventh TPR of the C-terminal TPR domain of Apc3, which interacts with another TPR-containing subunit, Apc8 (20) (Fig. 1A). Recent studies have shown that residues 362-368 (5th TPR) and 416 - 435 (7th TPR) of Apc7 interact with CBP/P300 (44) (Fig. 1A). Furthermore, several studies have shown that the C-terminal TPR domains of Apc7 and Apc3 contain regions for adaptor and substrate binding via an IR peptide (19) and that substrates (Nek2A) and adaptors (Cdc20) interact with APC/C simultaneously via IR peptides (45). Taken together, we suggest that the homodimeric conformation of Apc7 is more compatible with our U-shaped dimer model than the elongated dimer model (Fig. 8, $B$ and $C$ ).

The U-shaped model also explains how adaptor and substrate proteins that contain an IR peptide, such as NEK2A (NIMA (never in mitosis gene a)-related kinase 2) and cell division cycle 20 (45), are simultaneously recruited to $\mathrm{APC} / \mathrm{C}$, as well as the observation that the APC-C ${ }^{\mathrm{Cdc} 20}$ complex binds substrates that have higher affinities than either $\mathrm{APC} / \mathrm{C}$ or Cdc20 alone (46). In the U-shaped model, the two C-terminal TPR domains of an Apc7 dimer may contain binding sites for adaptors and substrates that contain an IR peptide. Presumably, the respective binding sites that are located in TPR motifs increase the efficiency of substrate binding to APC/C such that adaptors that are bound to TPR-containing subunits interact with incoming substrates and thereby guide the substrate IR peptide to its binding site (Fig. 8C).

Alternatively, simultaneous binding of adaptors and substrates also might be applicable to APC/C-mediated Cdc20 turnover, which is mediated independently of Cdh1 by the TPR-containing subunit Cdc27 (a homolog of Apc3) via the IR peptide (16). Furthermore, the U-shaped model may explain how the turnover of $\mathrm{Cdc} 20$ is mediated by neighboring $\mathrm{Cdc} 20$, which could play the role of an incoming substrate, like NEK2A. Further experimental validation, however, is needed to confirm the validity of the U-shaped model and to determine whether $\mathrm{Cdc} 20$ turnover is mediated by another $\mathrm{Cdc} 20$ molecule or directly by $\mathrm{APC} / \mathrm{C}$.

Consequently, the homodimeric conformation of Apc7 with its inherent flexibility is compatible with previous experimental findings. Although our proposed model must be validated in vivo and in vitro, our data on nAPc7 provide the first structural evidence of TPR-containing subunit assembly in APC/C.

\section{REFERENCES}

1. Peters, J. M. (2002) Mol. Cell 9, 931-943

2. Yoon, H. J., Feoktistova, A., Wolfe, B. A., Jennings, J. L., Link, A. J., and Gould, K. L. (2002) Curr. Biol. 12, 2048-2054

3. Harper, J. W., Burton, J. L., and Solomon, M. J. (2002) Genes Dev. 16, 2179-2206

4. Visintin, R., Prinz, S., and Amon, A. (1997) Science 278, 460 - 463

5. Glotzer, M., Murray, A. W., and Kirschner, M. W. (1991) Nature 349, $132-138$

6. Pfleger, C. M., and Kirschner, M. W. (2000) Genes Dev. 14, 655-665

7. Kraft, C., Vodermaier, H. C., Maurer-Stroh, S., Eisenhaber, F., and Peters, J. M. (2005) Mol. Cell 18, 543-553

8. Passmore, L. A., and Barford, D. (2004) Biochem. J. 379, 513-525

9. Peters, J. M. (2006) Nat. Rev. Mol. Cell Biol. 7, 644-656

10. Konishi, Y., Stegmüller, J., Matsuda, T., Bonni, S., and Bonni, A. (2004) Science 303, 1026-1030

11. van Roessel, P., Elliott, D. A., Robinson, I. M., Prokop, A., and Brand, A. H. (2004) Cell 119, 707-718

12. Gieffers, C., Dube, P., Harris, J. R., Stark, H., and Peters, J. M. (2001) Mol. Cell 7, 907-913

13. Dube, P., Herzog, F., Gieffers, C., Sander, B., Riedel, D., Müller, S. A., Engel, A., Peters, J. M., and Stark, H. (2005) Mol. Cell 20, 867-879

14. Passmore, L. A., Booth, C. R., Vénien-Bryan, C., Ludtke, S. J., Fioretto, C., Johnson, L. N., Chiu, W., and Barford, D. (2005) Mol. Cell 20, 855-866

15. Ohi, M. D., Feoktistova, A., Ren, L., Yip, C., Cheng, Y., Chen, J. S., Yoon, H. J., Wall, J. S., Huang, Z., Penczek, P. A., Gould, K. L., and Walz, T. (2007) Mol. Cell 28, 871-885

16. Thornton, B. R., Ng, T. M., Matyskiela, M. E., Carroll, C. W., Morgan, D. O., and Toczyski, D. P. (2006) Genes Dev. 20, 449-460

17. Wilson, C. G., Kajander, T., and Regan, L. (2005) FEBS J. 272, 166-179

18. Thornton, B. R., and Toczyski, D. P. (2006) Genes Dev. 20, 3069-3078

19. Vodermaier, H. C., Gieffers, C., Maurer-Stroh, S., Eisenhaber, F., and Peters, J. M. (2003) Curr. Biol. 13, 1459-1468

20. Lamb, J. R., Michaud, W. A., Sikorski, R. S., and Hieter, P. A. (1994) EMBO J. 13, 4321- 4328

21. Kraft, C., Herzog, F., Gieffers, C., Mechtler, K., Hagting, A., Pines, J., and Peters, J. M. (2003) EMBO J. 22, 6598-6609

22. Pál, M., Nagy, O., Ménesi, D., Udvardy, A., and Deák, P. (2007) J. Cell Sci. 120, $3238-3248$

23. Han, D., Kim, K., Oh, J., Park, J., and Kim, Y. (2008) Proteins 70, 900-914

24. Matthews, B. W. (1968) J. Mol. Biol. 33, 491-497

25. Gonzalez, A. (2003) Acta Crystallogr. Sect. D Biol. Crystallogr. 59, 1935-1942

26. Terwilliger, T. C. (2003) Methods Enzymol. 374, 22-37

27. Terwilliger, T. (2004) J. Synchrotron Radiat. 11, 49-52

28. Jones, T. A., Zou, J. Y., Cowan, S. W., and Kjeldgaard, M. (1991) Acta Crystallogr. Sect. A 47, 110-119

29. Emsley, P., and Cowtan, K. (2004) Acta Crystallogr. Sect. D Biol. Crystallogr. 60, 2126-2132

30. Winn, M. D., Isupov, M. N., and Murshudov, G. N. (2001) Acta Crystal- 


\section{Crystal Structure of the N-terminal Domain of Apc7}

logr. Sect. D Biol. Crystallogr. 57, 122-133

31. Painter, J., and Merritt, E. A. (2006) Acta Crystallogr. Sect. D Biol. Crystal$\log r$. 62, 439-450

32. Winn, M. D., Ashton, A. W., Briggs, P. J., Ballard, C. C., and Patel, P. (2002) Acta Crystallogr. Sect. D Biol. Crystallogr. 58, 1929-1936

33. Garnier, J., Gibrat, J. F., and Robson, B. (1996) Methods Enzymol. 266, $540-553$

34. Krissinel, E., and Henrick, K. (2004) Acta Crystallogr. Sect. D Biol. Crystallogr. 60, 2256-2268

35. Krissinel, E., and Henrick, K. (2007) J. Mol. Biol. 372, 774-797

36. Tanaka, T., Yokoyama, S., and Kuroda, Y. (2006) Biopolymers 84, 161-168

37. Thompson, J. D., Higgins, D. G., and Gibson, T. J. (1994) Nucleic Acids Res. 22, 4673-4680

38. Main, E. R., Xiong, Y., Cocco, M. J., D’Andrea, L., and Regan, L. (2003) Structure (Lond.) 11, 497-508
39. Holm, L., Kääriäinen, S., Rosenström, P., and Schenkel, A. (2008) Bioinformatics 24, $2780-2781$

40. Jínek, M., Rehwinkel, J., Lazarus, B. D., Izaurralde, E., Hanover, J. A., and Conti, E. (2004) Nat. Struct. Mol. Biol. 11, 1001-1007

41. Wu, Y., and Sha, B. (2006) Nat. Struct. Mol. Biol. 13, 589-593

42. Nyarko, A., Mosbahi, K., Rowe, A. J., Leech, A., Boter, M., Shirasu, K., and Kleanthous, C. (2007) Biochemistry 46, 11331-11341

43. Park, K. H., Choi, S. E., Eom, M., and Kang, Y. (2005) Breast Cancer Res. 7, R238-247

44. Turnell, A. S., Stewart, G. S., Grand, R. J., Rookes, S. M., Martin, A., Yamano, H., Elledge, S. J., and Gallimore, P. H. (2005) Nature 438, 690-695

45. Hayes, M. J., Kimata, Y., Wattam, S. L., Lindon, C., Mao, G., Yamano, H., and Fry, A. M. (2006) Nat. Cell Biol. 8, 607-614

46. Eytan, E., Moshe, Y., Braunstein, I., and Hershko, A. (2006) Proc. Natl. Acad. Sci. U. S. A. 103, 2081-2086 